\title{
Epidemiology of COVID-19
}

\author{
Sudipta Dhar Chowdhury, ${ }^{1,}$ Anu Mary Oommen²
}

\author{
${ }^{1}$ Department of Gastroenterology, Christian Medical College, \\ Vellore, India \\ 2Department of Community Medicine, Christian Medical College, \\ Vellore, India
}

J Digest Endosc 2020;11:3-7
Address for correspondence Sudipta Dhar Chowdhury, MD, DM, Department of Gastroenterology, Christian Medical College, Vellore 632004, India (e-mail: sudiptadharchowdhury@gmail.com).

\begin{abstract}
Keywords

- COVID-19

- coronavirus

- epidemiology

- pandemic

- SARS-CoV2

COVID-19, an infectious respiratory illness caused by the severe acute respiratory syndrome-corona virus 2 (SARS-CoV2), has now spread to multiple countries including India. The pace at which the disease spread in the last 4 months, since it was first recognized from China, is unprecedented. This review of the epidemiology of COVID-19 summarizes the burden of infection, transmission dynamics, and other related epidemiological features. While countries such as China, Italy, and the United States have particularly high-rates of infection, the disease is gradually spreading in India as well, threatening the health and economy of the country. Transmission in asymptomatic cases, early symptomatic phase, as well as limited access to testing in different settings are factors that have led to the rapid spread of infection. A large case series from China revealed that $81 \%$ of cases had mild symptoms, $14 \%$ had severe disease, and $5 \%$ were afflicted with critical illness. While the mortality in China was reported as $2.3 \%$, Italy, with a high-proportion of elderly, reported a case fatality report of $7.2 \%$ due to higher infection and mortality rates among the elderly. Being a highly infectious disease, with a basic reproduction number between 2 to 3, COVID-19 is affecting a large number of healthcare workers, as evidenced by the fact that a sizeable portion of reported infections in the US included healthcare workers. Delivering health care for both COVID-19 affected individuals, as well those with other acute and chronic conditions, with limited access to healthcare facilities and services, are challenges for the health systems in low- and middle-income countries, which require immediate measures for health system strengthening across sectors.
\end{abstract}

\section{Introduction}

Coronaviruses derive their name from the Latin word "corona" meaning crown. The name refers to the unique appearance of the virus under an electron microscope as round particles with a rim of projections resembling the solar corona. They are enveloped, positive-sense, single-stranded RNA viruses which were first isolated from humans in $1965 .{ }^{1}$ Coronavirus belongs to the family Coronaviridae which is known to produce mild respiratory diseases in humans. In recent times, there have been three major coronaviruses leading to disease outbreaks, beginning with the severe acute respiratory syndrome coronavirus (SARS-CoV) in 2002, followed by the
Middle East respiratory syndrome coronavirus (MERS-CoV) in 2012, and now the severe acute respiratory syndrome coronavirus 2 (SARS-CoV2).

\section{Disease Background}

In December 2019, China reported an outbreak of pneumonia of unknown causes in Wuhan, the capital city of Hubei province. Most of the early cases were epidemiologically linked to the Huanan seafood wholesale market where aquatic animals and live animals were sold. ${ }^{2}$ Using unbiased next-generation sequencing, an unknown betacoronavirus was discovered from lower respiratory tract samples of these patients. 
Human airway epithelial cells were used to isolate the virus that was named 2019-novel Coronavirus (2019-nCoV). ${ }^{3}$ The virus when observed under electron microscope had a diameter of 60 to $140 \mathrm{~nm}$ with characteristic spikes of 9 to $12 \mathrm{~nm}$, similar to the Coronoviridae family. ${ }^{3}$ Phylogenetically, the novel coronavirus was found to be more similar to two bat derived coronavirus strains ( $88 \%$ similarity) than coronaviruses which infect humans including SARS ( $79 \%$ similarity) and MERS ( $50 \%$ similarity).$^{4}$ Based on phylogeny and taxonomy on February 11, 2020, the Coronaviridae study group of the International Committee on Taxonomy of Viruses named the virus as SARS-CoV2. ${ }^{5}$ The World Health Organization (WHO) named the resultant disease as Coronavirus disease (COVID-19). ${ }^{6}$ On March 11, 2020, WHO, after assessing the situation across the globe, declared COVID-19 as a pandemic (-Fig. 1).

\section{Geographic Distribution}

Since the initial report from China, the disease spread rapidly, and the number of cases increased exponentially. On January 11 , the first case was reported outside mainland China in Thailand, ${ }^{7}$ and within months, the disease spread to all the continents except Antarctica. India reported its first case of COVID-19 on January 30, 2020. This rose to three cases by February 3, 2020. No further cases were reported in February 2020. However, by mid-March, the number of infected cases started to increase, and many cases were reported from all over India. The first COVID-19 related death in India was reported on March 12, 2020. By the second week of April, the disease spread to all states in India except Sikkim. At the time of writing this manuscript, there have been 2,170,265 cases and 135,163 deaths globally and 15,712 cases and 507 deaths in India.,

\section{Transmission}

Zoonotic transmission initially appeared to be a plausible cause as majority of early cases had a history of exposure to wet markets. ${ }^{2}$ However, by the end of January 2020, the number of people who developed the disease without exposure to the market or another person with respiratory symptoms increased. The spread of the disease among persons who did not visit Wuhan and among healthcare workers suggested a person-to-person spread of the virus. ${ }^{10,11}$ The exact mode of transmission of this virus is unknown. But, as with other respiratory viruses, droplet borne infection, either directly or indirectly, through fomites is probably the predominant mode of transmission. At present, there is no evidence for airborne transmission of the virus. ${ }^{12,13}$ Although virus particles have been detected in stool samples of both symptomatic and convalescing patients, the risk of feco-oral transmission is unclear. ${ }^{14,15}$

\section{Infectiousness/Transmissibility of SARS-CoV2}

The basic reproduction number $\left(R_{0}\right)$ is the expected number of secondary cases that could arise from one case in a susceptible population. $\mathrm{R}_{0}$ is the essence of infectious disease epidemiology and indicates the risk of an epidemic spread. Most studies have estimated the $\mathrm{R}_{0}$ for SARS-CoV2 to be within a range of 2.0 to 3.0. ${ }^{16}$ The other parameter which determines infectivity is the secondary attack rate. It is defined as the probability of an infection occurring in a specific group of susceptible people exposed to a primary case (e.g., household contacts or close contacts). The secondary attack rate provides an insight into how social behaviors affects transmissibility. The secondary attack rates among household contacts was similar in South Korea and the United States (7.5\% and $10.5 \%$, respectively). ${ }^{17,18}$

\section{Incubation Period and Serial Interval}

The mean or median incubation period of the disease ranges from 5 to 6 days. ${ }^{19-21}$ Lauer et al estimated that 2.5\% of the patients will develop symptoms within 2.2 days $(95 \% \mathrm{CI}$, 1.8 to 2.9 days) and $97.5 \%$ of patients will develop symptoms within 11.5 days ( $95 \% \mathrm{CI}, 8.2-15.6$ days). ${ }^{19}$

Serial interval refers to the time interval between the onset of symptoms in the primary case and the secondary case. The mean serial interval is estimated to be approximately 4

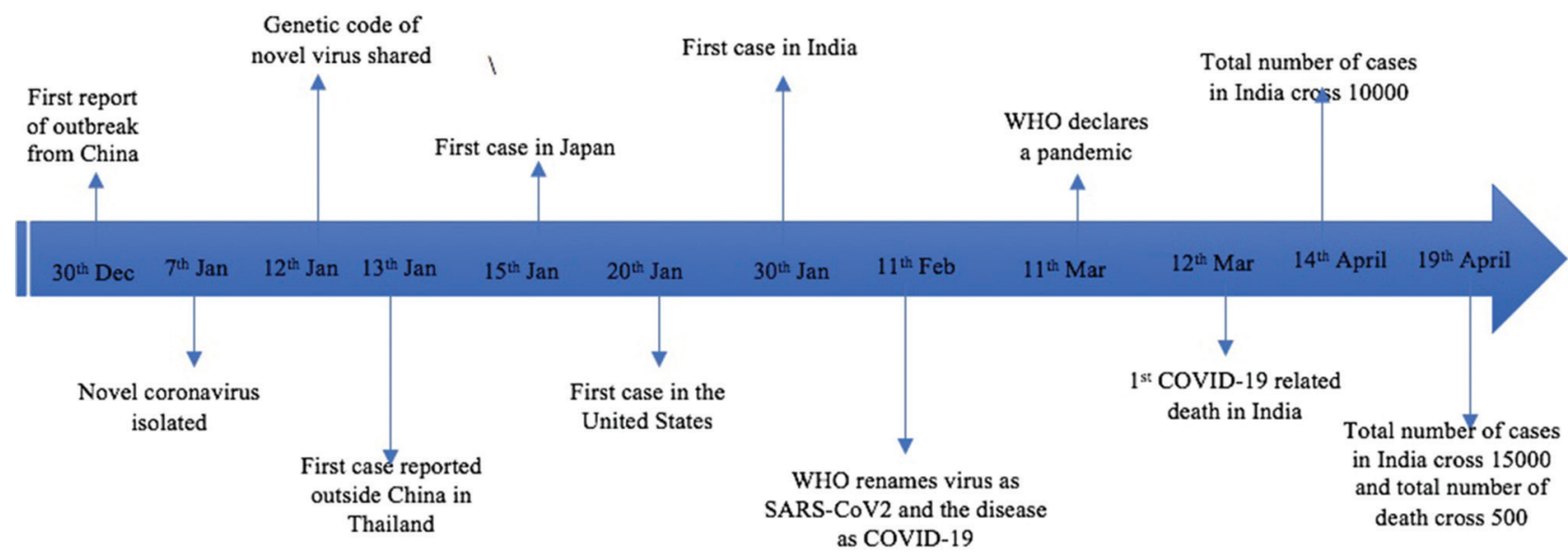

Fig. 1 Timeline of important events between December 2019 to April 2020. 
to 5 days. ${ }^{22,23}$ By analyzing data from 468 infector-infectee pairs, Du et al noted that 59 secondary cases had symptoms earlier than their primary case. This suggested that there is a possibility that the transmission of the disease occurred during the asymptomatic phase of illness in this group of patients. ${ }^{24}$

\section{Period of Infectivity}

The duration for which a patient with COVID-19 remains infective is unclear. Viral load in the oropharyngeal secretions is highest during the early symptomatic stage of the disease. ${ }^{25,26}$ The patient can continue to shed the virus even after symptom resolution. ${ }^{26}$ In a study from China, the median duration of virus shedding was 20 days (interquartile range [IQR] 17.0-24.0) amongst the survivors. ${ }^{27}$ A study of viral dynamics in mild and severe cases revealed that mild cases tend to clear the viruses early, while severe cases can have prolonged viral shedding. ${ }^{28}$ Data from studies using twin respiratory and fecal sampling have shown viral shedding can persist in stools for more than 4 weeks even when respiratory samples are negative..$^{29} \mathrm{Xu}$ et al identified male sex, delayed hospitalization after illness, and invasive mechanical ventilation as risk factors for prolonged viral shedding. ${ }^{30}$ Transmission during the asymptomatic phase has also been reported. In a study from Singapore, 6.4\% of the 157 locally acquired cases of COVID-19 were attributed to transmission during the asymptomatic phase of the disease. ${ }^{31}$

\section{Clinical Features}

The spectrum of COVID 19 disease ranges from asymptomatic to critical including mortality. In a report on 72314 cases from the Chinese Center for Disease Control and Prevention, $81 \%$ cases were classified as mild (no pneumonia or mild pneumonia), $14 \%$ as severe (14\%; dyspnea, respiratory frequency $\leq 30 / \mathrm{min}$, blood oxygen saturation $<93 \%$, PF ratio $<300$, and or lung infiltrated $>50 \%$ within $24-48$ hours), and $5 \%$ as critical (respiratory failure, septic shock, and/or multiorgan failure). ${ }^{32}$

\section{Clinical Manifestations}

Fever is probably one of the most common manifestations of the disease. In a large study from China, although fever was present in $44 \%$ of patients at admission, but after admission, $88 \%$ developed fever. ${ }^{33} \mathrm{~A}$ dry cough is reported in approximately 65 to $70 \%$ of patients. Among patients from Europe, anosmia or hyposmia and loss of taste have been reported in $85 \%$ and $88 \%$, respectively. ${ }^{34}$ Anorexia is also a frequent complaint. Myalgia and dyspnea were seen in approximately $30 \%$ of patients. ${ }^{35}$ In a meta-analysis of 60 studies including 4243 patients, the prevalence of gastrointestinal symptoms was $17.6 \%$ (95 CI 12.3-24.5\%). Gastrointestinal symptoms included diarrhea, abdominal pain, and vomiting/nausea in $13 \%, 9 \%$, and $10 \%$ respectively. ${ }^{36}$

The clinical course of the disease varies widely. In the study of Wang et al, the median time from the first symptom to dyspnea was 5 days, to hospitalization 7 days, and acute respiratory distress syndrome (ARDS) 8 days. ICU admission was required for patients who developed ARDS (61\%), arrhythmia (44\%), or shock (30\%). Patients treated in ICU are older (median age 66 years) and more likely to have comorbidities $(72.2 \%)^{35}$

\section{Risk Factors Associated with Adverse Outcome}

Although the disease can affect any age group, the elderly and patients with comorbidities are at risk for severe disease. Data from the China and the United States indicate that the hospitalization rates, ICU admissions, and mortality rates are higher in older adults. ${ }^{32,37}$ The Chinese Center for Disease Control and Prevention identified comorbidities such as cardiovascular disease, diabetes mellitus, chronic respiratory disease, hypertension, and cancer to be associated with adverse outcome. ${ }^{32}$

\section{Case Fatality Rate}

Case fatality rate (CFR) is the proportion of deaths from a certain disease among the total number of people affected by the disease within a certain period of time and is a reflection of the virulence of the infective organism.

In the report from the Chinese Center for Disease Control and Prevention, the overall CFR was 2.3\% (1023 deaths among 44672 confirmed cases). ${ }^{32}$ Compared with China, the CFR was higher in Italy $7.2 \%{ }^{38}$ The high CFR in Italy is probably due to the higher rate of infections among the elderly (proportion of cases $\geq 70$ year of all cases: 37 vs. $11 \%$ in Italy and China, respectively). The age-specific CFR were similar between China and Italy in the age groups below 70 years. But in age groups between 70 to 79 years and $\geq 80$ years, the CFR was higher in Italy ( $12.8 \%$ and $20.2 \%$, respectively) than China ( $8 \%$ and $14.8 \%$, respectively). In the Italian cohort, 687 patients were $\geq 90$ years, and in them, the CFR was $22 \%{ }^{38}$

\section{Immunity}

As SARS-CoV2 is a novel virus, the entire global population is susceptible to the virus. The population will continue to remain at risk until and unless there is the development of herd immunity either through vaccination or infection. Herd immunity is an indirect form of immunity that develops when a large percentage of the population develops immunity to the infection either through infection or vaccination, thereby limiting the spread of the virus. $\mathrm{R}_{0}$ determines the percentage of the population which needs to be immune to achieve herd immunity. Using an estimated $\mathrm{R}_{0}$ of 3 , the estimated proportion of the world's population that needs to develop immunity to SAR-CoV2 to develop herd immunity is approximately $70 \%{ }^{39}$ Considering the risk of severe disease in a significant number, herd immunity would preferably be best obtained through vaccination. The WHO is coordinating with a group of experts for vaccine development. ${ }^{40}$ As on 20th April 2020, there are 5 candidate vaccines under clinical evaluation. Of the five, one is in Phase 2 trial while the other four are in Phase 1 trial. Seventy-one candidate vaccines are undergoing preclinical evaluation. ${ }^{41}$ 


\section{COVID-19 and Healthcare Workers}

As the pandemic progresses, healthcare systems all over the world are being challenged. In China, more than 3300 healthcare workers (HCWs) have been affected and many have died. ${ }^{42}$ Among the 49,370 COVID-19 cases reported to CDC between February 12 to April 9, 2020, from all states in the Unites States, 9282 (19\%) are HCWs. Most (90\%) of the HCWs affected with COVID-19 did not require hospitalization; however, 27 deaths were recorded across all age groups. ${ }^{43}$ This reflects the need to protect HCWs in this pandemic, as they are probably the most at-risk population for contracting the disease.

\section{Prevention}

COVID-19 is an infectious disease, posing a massive challenge to global health, which will probably run a prolonged course till an effective vaccine is found, or herd immunity is achieved. Until availability of effective vaccines for a large percentage of the global population, public health measures such as social distancing, isolation, and quarantine are needed to prevent the spread of the virus. WHO recommends protective measures such as frequent hand washing, avoiding touching of eyes, nose or mouth, maintaining a distance of at least $1 \mathrm{~m}$ between people, and practicing respiratory hygiene, to help prevent the spread of the disease ${ }^{44}$

HCWs and health administrators as frontline defence in this battle against COVID-19 need to be geared up for a prolonged battle. In low- and middle-income countries, like India, additional health system challenges include the large numbers affected by chronic conditions that predispose to severe illness, poor control of chronic conditions, difficulties in accessing healthcare due to lack of adequate, and equitably distributed healthcare services, which are now compounded by the loss of income and mobility with containment measures such as the nationwide lockdown. Strategies to increase universal health coverage, with massive infrastructural changes, upgradation of the public healthcare systems, and strategic involvement of the private health sector are required to rise up to this novel challenge that the virus poses, with lasting benefits for both the current epidemic and future health challenges.

\section{Funding}

None.

\section{Conflicts of Interest}

None declared.

\section{References}

1 Tyrrell DA, Bynoe ML. Cultivation of viruses from a high proportion of patients with colds. Lancet 1966;1(7428):76-77

2 Li Q Guan X, Wu P, et al. Early transmission dynamics in Wuhan, China, of novel Coronavirus-infected pneumonia. N Engl J Med 2020;382(13):1199-1207

3 Zhu N, Zhang D, Wang W, et al; China Novel Coronavirus Investigating and Research Team. A novel Coronavirus from patients with pneumonia in China, 2019. N Engl J Med 2020; 382(8):727-733

4 Lu R, Zhao X, Li J, et al. Genomic characterisation and epidemiology of 2019 novel coronavirus: implications for virus origins and receptor binding. Lancet 2020;395(10224):565-574

5 Coronaviridae Study Group of the International Committee on Taxonomy of VirusesNature Microbiology. The species Severe acute respiratory syndrome-related coronavirus: classifying 2019-nCoV and naming it SARS-CoV-2. 2020;(e-pub ahead of print). doi https://www.ncbi.nlm.nih.gov/pmc/articles/ PMC7095448

6 WHO. Naming the coronavirus disease (COVID-19) and the virus that causes it. Available at: https://www.who.int/emergencies/diseases/novel-coronavirus-2019/technical-guidance/ naming-the-coronavirus-disease-(covid-2019)-and-the-virus-that-causes-it. Accessed April 16, 2020

7 WHO statement on novel coronavirus in Thailand. Available at: https://www.who.int/news-room/detail/13-01-2020-whostatement-on-novel-coronavirus-in-thailand. Accessed April 16,2020

8 WHO. COVID-19 Dashboard. Available at: https://covid19. who.int/. Accessed April 17, 2020

9 Ministry of Health and Family Welfare. Available at: Https:// Www.Mohfw.Gov.In/ Accessed April 23, 2020

10 Ralph R, Lew J, Zeng T, et al. 2019-nCoV (Wuhan virus), a novel Coronavirus: human-to-human transmission, travel-related cases, and vaccine readiness. J Infect Dev Ctries 2020;14(1):3-17

11 Chan JF-W, Yuan S, Kok K-H, et al. A familial cluster of pneumonia associated with the 2019 novel Coronavirus indicating person-to-person transmission: a study of a family cluster. Lancet 2020;395(10223):514-523

12 Faridi S, Niazi S, Sadeghi K, et al. A field indoor air measurement of SARS-CoV-2 in the patient rooms of the largest hospital in Iran. Sci Total Environ 2020;725:138401

13 Wong SC-Y, Kwong RT-S, Wu TC, et al. Risk of nosocomial transmission of coronavirus disease 2019: an experience in a general ward setting in Hong Kong. J Hosp Infect 2020;(April) :S0195-6701(20)30174-2

14 Chen Y, Chen L, Deng Q, et al. The presence of SARS-CoV-2 RNA in feces of COVID-19 patients. J Med Virol 2020;(April) doi 10.1002/jmv.25825

15 Liu J, Xiao Y, Shen Y, et al. Detection of SARS-CoV-2 by RT-PCR in anal from patients who have recovered from coronavirus disease 2019. J Med Virol 2020;(April) doi 10.1002/jmv.25875

16 Park M, Cook AR, Lim JT, Sun Y, Dickens BL. A systematic review of COVID-19 epidemiology based on current evidence. J Clin Med 2020;9(4):E967

17 COVID-19 National Emergency Response Center, Epidemiology and Case Management Team, Korea Centers for Disease Control and Prevention. Coronavirus disease-19: summary of 2,370 contact investigations of the first 30 cases in the Republic of Korea. Osong Public Health Res Perspect 2020;11(2):81-84

18 Burke RM, Midgley CM, Dratch A, et al. Active monitoring of persons exposed to patients with confirmed COVID-19 - United States, January-February 2020. MMWR Morb Mortal Wkly Rep 2020;69(9):245-246

19 Lauer SA, Grantz KH, Bi Q et al. The incubation period of Coronavirus disease 2019 (COVID-19) from publicly reported confirmed cases: estimation and application. Ann Intern Med 2020;(e-pub ahead of print). doi 10.7326/M20-0504

20 Linton NM, Kobayashi T, Yang Y, et al. Incubation period and other epidemiological characteristics of 2019 novel Coronavirus infections with right truncation: a statistical analysis of publicly available case data. J Clin Med 2020;9(2):E538

21 Backer JA, Klinkenberg D, Wallinga J. Incubation period of 2019 novel coronavirus (2019-nCoV) infections among 
travellers from Wuhan, China, 20-28 January 2020. Euro Surveill 2020;25(5doi 10.2807/1560-7917.ES.2020.25.5.2000062

22 Nishiura H, Linton NM, Akhmetzhanov AR. Serial interval of novel coronavirus (COVID-19) infections. Int J Infect Dis 2020;93:284-286

23 Zhang J, Litvinova M, Wang W, et al. Evolving epidemiology and transmission dynamics of coronavirus disease 2019 outside Hubei province, China: a descriptive and modelling study. Lancet Infect Dis 2020;(April) :S1473-3099(20)30230-9

24 Du Z, Xu X, Wu Y, Wang L, Cowling BJ, Meyers LA. Serial interval of COVID-19 among publicly reported confirmed cases. Emerg Infect Dis 2020;26(6doi 10.3201/eid2606.200357

25 To KK-W, Tsang OT-Y, Leung W-S, et al. Temporal profiles of viral load in posterior oropharyngeal saliva samples and serum antibody responses during infection by SARS-CoV-2: an observational cohort study. Lancet Infect Dis 2020;(March) :S1473-3099(20)30196-1

26 Wölfel R, Corman VM, Guggemos W, et al. Virological assessment of hospitalized patients with COVID-2019. Nature 2020;(e-pub ahead of print). doi 10.1038/s41586-020-2196-X

27 Zhou F, Yu T, Du R, et al. Clinical course and risk factors for mortality of adult inpatients with COVID-19 in Wuhan, China: a retrospective cohort study. Lancet 2020;395(10229) :1054-1062

28 Liu Y, Yan L-M, Wan L, et al. Viral dynamics in mild and severe cases of COVID-19. Lancet Infect Dis 2020;(March) :S1473-3099(20)30232-2

$29 \mathrm{Wu}$ Y, Guo C, Tang L, et al. Prolonged presence of SARS-CoV-2 viral RNA in faecal samples. Lancet Gastroenterol Hepatol 2020;5(5):434-435

$30 \mathrm{Xu} \mathrm{K}$, Chen Y, Yuan J, et al. Factors associated with prolonged viral RNA shedding in patients with COVID-19. Clin Infect Dis 2020;(e-pub ahead of print). doi 10.1093/cid/ciaa351

31 Wei WE, Li Z, Chiew CJ, Yong SE, Toh MP, Lee VJ. Presymptomatic transmission of SARS-CoV-2 - Singapore, January 23-March 16, 2020. MMWR Morb Mortal Wkly Rep 2020;69(14):411-415

$32 \mathrm{Wu}$ Z, McGoogan JM. Characteristics of and important lessons from the Coronavirus disease 2019 (COVID-19) outbreak in China: summary of a report of 72314 cases from the Chinese Center for Disease Control and Prevention. JAMA 2020;(e-pub ahead of print). doi 10.1001/jama.2020.2648

33 Guan W-J, Ni Z-Y, Hu Y, et al; China Medical Treatment Expert Group for COVID-19. Clinical characteristics of Coronavirus disease 2019 in China. N Engl J Med 2020;(e-pub ahead of print). doi 10.1056/NEJMoa2002032

34 Lechien JR, Chiesa-Estomba CM, De Siati DR, et al. Olfactory and gustatory dysfunctions as a clinical presentation of mild-to-moderate forms of the coronavirus disease (COVID19): a multicenter European study. Eur Arch Otorhinolaryngol 2020;(e-pub ahead of print). doi 10.1007/s00405-020-05965-1

35 Wang D, Hu B, Hu C, et al. Clinical characteristics of 138 hospitalized patients with 2019 novel Coronavirus-infected pneumonia in Wuhan, China. JAMA 2020;(e-pub ahead of print). doi 10.1001/jama.2020.1585

36 Cheung KS, Hung IF, Chan PP, et al. Gastrointestinal manifestations of SARS-CoV-2 infection and virus load in fecal samples from the Hong Kong Cohort and systematic review and meta-analysis. Gastroenterology 2020;(April) :S0016-5085(20)30448-0

37 CDC COVID-19 Response Team. Severe outcomes among patients with Coronavirus disease 2019 (COVID-19) - United States, February 12-March 16, 2020. MMWR Morb Mortal Wkly Rep 2020;69(12):343-346

38 Onder G, Rezza G, Brusaferro S. Case-fatality rate and characteristics of patients dying in relation to COVID-19 in Italy. JAMA 2020;(e-pub ahead of print). doi 10.1001/jama.2020.4683

39 Syal K. COVID-19: herd immunity and convalescent plasma transfer therapy. J Med Virol 2020;(e-pub ahead of print). doi 10.1002/jmv.25870

40 Public statement for collaboration on COVID-19 vaccine development. https://www.who.int/news-room/detail/13-042020-public-statement-for-collaboration-on-covid-19-vaccine-development. Accessed April 29, 2020.

41 www.who.int/blueprint/priority-diseases/key-action/novel-coronavirus-landscape-ncov.pdf

42 The Lancet. COVID-19: protecting health-care workers. Lancet Lond Engl. 2020;395(10228):922. doi:10.1016/S01406736(20)30644-9

43 CDC COVID-19 Response Team. Characteristics of Health Care Personnel with COVID-19 - United States, February 12-April 9, 2020. MMWR Morb Mortal Wkly Rep. 2020;69(15):477-481. doi:10.15585/mmwr.mm6915e6

44 Advice for public. https://www.who.int/emergencies/diseases/ novel-coronavirus-2019/advice-for-public. Accessed April 18, 2020. 